\title{
HAMILTONIAN ANALYSIS OF THE GENERALIZED PROBLEM OF BOLZA
}

\author{
F. H. CLARKE
}

\begin{abstract}
RESUME. On étudie le problème généralisé de Bolza en calcul des variations. Presented at the International Conference on the Calculus of Variations held to honour the memory of Leonida Tonelli, Scuola Normale Superiore, Pisa, March 1986. On obtient des conditions nécessaires en forme hamiltonienne, sous des hypothèses moins exigeantes qu'antérieurement, en particulier sans qualification sur les contraintes. Le lien avec les problèmes de contrôle optimal est développé, ainsi que l'apport de ces conditions à la théorie de la régularité de la solution.
\end{abstract}

\begin{abstract}
We obtain necessary conditions in Hamiltonian form for the generalized problem of Bolza in the calculus of variations. These are proven in part by an extension to Hamiltonians of Tonelli's method of auxiliary Lagrangians. One version of the conditions is of a new character since it is obtained in the absence of any constraint qualification on the data. A new regularity theorem is shown to be a consequence of the necessary conditions.
\end{abstract}

1. Introduction. The generalized problem of Bolza consists of minimizing the functional $J$ defined by

$$
J(x):=f(x(b))+\int_{a}^{b} L\left(t, x(t), x^{\prime}(t)\right) d t
$$

over the $\operatorname{arcs} x$ (i.e., absolutely continuous functions from $[a, b]$ to $R^{n}$ ) satisfying $x(a) \in C, x(b) \in D$. What distinguishes this from a classical problem of Bolza in the calculus of variations is the lack of regularity assumptions, and especially the fact that the Lagrangian $L$ is allowed to be extended-valued (i.e., to assume the value $+\infty$ ). The fact that at this level of generality the problem subsumes a variety of constrained variational problems and problems of optimal control has been extensively studied and is now well known (see for example $[\mathbf{1}, \mathbf{3}, \mathbf{6}]$ ).

An important theme in the study of the problem has been the attempt to analyse it as much as possible through the Hamiltonian, the function $H$ defined by

$$
H(t, x, p):=\sup \left\{\langle p, v\rangle-L(t, x, v): v \in R^{n}\right\} .
$$

The reason in part is based upon the hope that for certain classes of problems for which $L$ is intractable, $H$ may actually be relatively well behaved. In his cogent

Received by the editors June 11, 1986. Presented at the International Conference on the Calculus of Variations held to honour the memory of Leonida Tonelli, Scuola Normale Superiore, Pisa, March 1986.

1980 Mathematics Subject Classification (1985 Revision). Primary 49B05.

Key words and phrases. Bolza problem, calculus of variations, Hamiltonian, necessary conditions, nonsmooth analysis, generalized gradients, regularity.

Ce rapport a été publié en partie grâce à une subvention du Fonds FCAR pour l'aide et le soutien à la recherche. 
article, Rockafellar [6] carried out this program as regards the issue of existence. Necessary conditions for optimality were broached by Clarke [2], and sufficiency was addressed by Zeidan [8]. The hypotheses employed in the necessary conditions were primarily the "strong Lipschitz condition" on $H$, and a constraint qualification ("calmness") defined in terms of the asymptotic behavior of the problem under perturbation of the endpoint constraints. While they are appropriate for several classes of problems that arise, these hypotheses are somewhat restrictive, and one of our purposes here is to relax them substantially. In particular, we obtain for the first time necessary conditions for the generalized problem of Bolza in the absence of any constraint qualification whatever.

In a recent article, Clarke and Vinter [5] obtain substantial new results on the regularity of the solution to variational problems in which the Lagrangian is well behaved (for example, locally Lipschitz, or continuously differentiable). They employ in part a technique of "auxiliary Lagrangians" inspired by Tonelli's work. The present article extends some of their results to the significantly broader context of the generalized problem (which includes variational problems with various side constraints as special cases). In view of the program stated above, it is fitting that one of the techniques employed is that of "auxiliary Hamiltonians".

$\S \S 2$ and 3 state and prove the principal necessary conditions (Theorem 2.1) in the presence of calmness. In $\S 4$, we show how to combine these conditions with Rockafellar's theory of proximal subgradients to treat the problem in the absence of the calmness hypothesis, and we specialize the results to two important special cases.

2. Necessary conditions in the calm case. We consider the problem of Bolza described in the introduction, that of minimizing the functional $J(x)$ over all $\operatorname{arcs} x$ satisfying $x(a) \in C, x(b) \in D$. The basic technical hypotheses (present at all times) are that $C$ and $D$ are closed, that $f$ is locally Lipschitz, and that the Lagrangian

$$
L(t, x, v):[a, b] \times R^{n} \times R^{n} \rightarrow R \cup\{+\infty\}
$$

is $L \times B$-measurable, lower semicontinuous in $(x, v)$ and convex as a function of $v$. (The measurability requirement means that $L$ is measurable with respect to the $\sigma$-field generated by products of Lebesgue subsets of $[a, b]$ and Borel subsets of $R^{n} \times R^{n}$.)

Now let the arc $z$ be a (strong) local solution to this problem. This means that for some $\varepsilon_{0}>0$, for some set $\Omega$ given by

$$
\Omega=\left\{(t, y) \in[a, b] \times R^{n}:|y-z(t)| \leq \varepsilon_{0}\right\},
$$

we have $J(z) \leq J(x)$ whenever $x$ is an arc satisfying the boundary constraints as well as

$$
(t, x(t)) \in \Omega \quad \text { for all } t \in[a, b] .
$$

Let us redefine $L(t, x, v)$ as $+\infty$ for $|x-z(t)|>\varepsilon_{0}$, which affects neither the hypotheses nor the conclusion of the theorem while rendering $z$ a global solution. For clarity, we shall also retain the explicit constraint $(t, x(t)) \in \Omega$. With a slight abuse of notation, an arc $x$ satisfying this constraint (for all $t$ in $[a, b]$ ) is said to lie in $\Omega$.

Let $H$ be the Hamiltonian corresponding to $L$ as defined in the introduction. $H$ is said to satisfy the basic growth condition (see Rockafellar [6]) provided that for 
every bounded subset $S$ of $R^{n}$ and for each fixed $p \in R^{n}$ there exists a summable function $\varphi:[a, b] \rightarrow R$ such that

$$
H(t, x, p) \leq \varphi(t) \quad \text { for all }(t, x) \in[a, b] \times S .
$$

This mild condition generalizes classical ones employed in existence theory by Nagumo and Tonelli and later by Cesari and Olech (see [6]).

We designate by $A$ the Banach space of arcs endowed with the norm

$$
\|x\|_{a}:=|x(a)|+\int_{a}^{b}\left|x^{\prime}(t)\right| d t .
$$

(Clearly $A$ is isomorphic to $R^{n} \times L^{1}[a, b]$.) $C$ designates the Banach space of continuous functions $x$ from $[a, b]$ to $R^{n}$ endowed with the norm

$$
\|x\|_{\infty}:=\max _{a \leq t \leq b}|x(t)| \text {. }
$$

The following result $[6]$ plays a central role in this article.

ROCKAFELLAR'S SEMICONTINUITY THEOREM. Let $H$ satisfy the basic growth condition on $\Omega$. Then for all real numbers $\alpha$ and $r$ the set $\{x \in A: J(x) \leq$ $\left.\alpha,\|x\|_{\infty} \leq r\right\}$ is compact in the weak topology of $A$ and also in the strong topology of $C$. In particular, $J(\cdot)$ is lower semicontinuous relative to the norms of $A$ and $C$ and lower semicontinuous sequentially relative to the weak topology of $A$.

An immediate consequence of the theorem is that $J$ admits a minimizing arc whenever there is a minimizing sequence which is bounded in the supremum norm. (Note that in our present setting $\|x\|_{\infty}$ is always bounded if $J(x)$ is finite, since $L(t, x, v)$ is $+\infty$ for $(t, x)$ lying outside the compact set $\Omega$.)

We now define, for any positive $M$,

$$
h(t, M):=\sup \{H(t, y, p):(t, y) \in \Omega,|p| \leq M\} .
$$

Rockafellar [6] proves that (under the basic growth condition), $h(\cdot, M)$ is integrable for each $M$, and that $H(t, y, p)$ is $L \times B$-measurable and upper semicontinuous in $(y, p)[6$, Proposition 4]. In what follows, $\partial H$ shall always refer to the generalized gradient taken with respect to the $(y, p)$ variables. We next assume the following weak Lipschitz condition:

For certain constants $c_{1}, c_{2}$ and for some integrable function $k_{1}(t)$ on $[a, b]$, the following holds:

$$
|\varphi| \leq\{1+|p|\}\left\{k_{1}(t)+c_{1}|\psi|\right\}+c_{2}|H(t, y, p)|
$$

for all $(\varphi, \psi)$ in $\partial H(t, y, p)$, for all $(t, y)$ in the interior of $\Omega$, for all $p$.

When the constants $c_{1}$ and $c_{2}$ of (H1) are required to be zero, we obtain the strong Lipschitz condition of [2]; note that if $H$ were a smooth function, the key inequality of (H1) would reduce to

$$
\left|H_{x}\right| \leq\{1+|p|\}\left\{k_{1}(t)+c_{1}\left|H_{p}\right|\right\}+c_{2}|H| .
$$

In analogy with $h$ we proceed to define

$$
\begin{aligned}
& h_{x}(t, M):=\sup \left\{|\varphi|: \varphi \in \partial_{x} H(t, y, p),(t, y) \in \operatorname{Int} \Omega,|p| \leq M\right\}, \\
& h_{p}(t, M):=\sup \left\{|\psi|: \psi \in \partial_{p} H(t, y, p),(t, y) \in \operatorname{Int} \Omega,|p| \leq M\right\} .
\end{aligned}
$$


The following imposes an integrability condition in $t$ of the data

$$
\text { for each } M, h_{x}(\cdot, M) \text { and } h_{p}(\cdot, M) \text { lie in } L^{1}[a, b] .
$$

In the presence of the basic growth condition, it follows from (H2) that for almost each $t, H(t, \cdot, \cdot)$ is locally Lipschitz on Int $\Omega \times R^{n}$, and that the Lipschitz rank is integrable in $t$ for $p$ restricted to bounded sets. Conversely, if for every bounded subset $S$ of $R^{n}, H(t, \cdot, \cdot)$ is Lipschitz on Int $\Omega \times S$ with Lipschitz constant $k(t)$ such that $k(\cdot)$ lies in $L^{1}[a, b]$, then $(\mathrm{H} 2)$ is true. This equivalence makes clearer the meaning of hypothesis (H2), and demonstrates that it holds automatically if $H$ is a locally Lipschitz function of $(x, p)$ not depending on $t$ (i.e., if the problem is autonomous).

Let us now define the problem (P) as that of minimizing $J(x)$ over the arcs $x$ lying in $\Omega$ and satisfying $x(a) \in C, x(b) \in D$. We imbed $(\mathrm{P})$ in a family of problems $\left(\mathrm{P}_{\alpha}\right)$, where for each $\alpha$ in $R^{n},\left(\mathrm{P}_{\alpha}\right)$ is the problem of minimizing $J(x)$ over the arcs $x$ in $\Omega$ satisfying $x(a) \in C, x(b) \in D+\alpha$. We define $V(\alpha)$ to be the infimum in $\left(\mathrm{P}_{\alpha}\right)$; thus $V(0)=J(z)$.

(P) is said to be calm provided that we have

$$
\liminf _{\alpha \rightarrow 0} \frac{V(\alpha)-V(0)}{|\alpha|}>-\infty .
$$

Note that if $D=R^{n}$ then $(\mathrm{P})$ is automatically calm, since $\left(\mathrm{P}_{\alpha}\right)$ and $(\mathrm{P})$ coincide, so that $V$ is constant.

We denote by $\operatorname{cl} N_{C}$ the multifunction whose graph is the closure of that of $N_{C}$. It is known that $\operatorname{cl} N_{C}(x)$ agrees with the usual normal cone $[3] N_{C}(x)$ under mild regularity hypotheses, for example if $C$ is convex or defined classically by (nondegenerate) equalities and/or inequalities, or if the tangent cone $T_{C}(x)$ has nonempty interior.

To summarize, the hypotheses are the basic technical ones, the basic growth condition, calmness, and (H1)-(H2).

THEOREM 2.1. Under the hypotheses above, the solution $z$ admits an arc $p$ on $[a, b]$ such that $\left(-p^{\prime}(t), z^{\prime}(t)\right) \in \partial H(t, z(t), p(t))$ a.e., $p(a) \in \operatorname{cl} N_{C}(z(a)),-p(b) \in$ $\partial f(z(b))+N_{D}(z(b))$.

REMARK. It is possible to work within the framework of the Bolza problem treated in $[\mathbf{2}]$, which involves the minimization of

$$
K(x):=\Lambda(x(a), x(b))+\int_{a}^{b} L\left(t, x(t), x^{\prime}(t)\right) d t
$$

where $\Lambda$ is lower semicontinuous and extended-valued. This appears more general than the problem we have been considering, since $\Lambda$ may be taken to be $f(x(b))$ when $(x(a), x(b))$ lies in $C \times D$ and $+\infty$ otherwise, but a passage in the other direction is also possible. To effect it, we extend the state $x$ in $R^{n}$ to a state $(y, x, r)$ in $R^{n} \times R^{n} \times R$, and we define a new Lagrangian $M(t, y, x, r, w, v, s)$ to be equal to $L(t, x, v)$ if $w$ and $s$ are 0 , and $+\infty$ otherwise. We also define

$$
C=\{(y, x, r): y=x\}, \quad D=\operatorname{epi}(\Lambda), \quad f(y, x, r)=r .
$$

It is routine to verify that the extended arc $(z(a), z(t), \Lambda(z(a), z(b)))$ solves this new problem, and that its Lagrangian and Hamiltonian satisfy the hypotheses of the 
theorem if the original data do. The calmness of the extended problem is equivalent to the condition

$$
\liminf _{(\alpha, \beta) \rightarrow(0,0)} \frac{W(\alpha, \beta)-W(0,0)}{|(\alpha, \beta)|}>-\infty
$$

where $W(\alpha, \beta)$ is defined to be the infimum over $x$ of the perturbed functional

$$
\Lambda(x(a)+\alpha, x(b)+\beta)+\int_{a}^{b} L\left(t, x(t), x^{\prime}(t)\right) d t .
$$

In this context the Hamiltonian inclusion of the theorem is joined by the transversality condition $(p(a),-p(b)) \in \partial \Lambda(z(a)), z(b))$. This condition may be more precise than that of the theorem when $\Lambda$ stems from $f, C$ and $D$ as indicated above, to the extent that $N_{C}$ fails to be closed at $z(a)$; the price however is that a joint calmness condition in both endpoints is needed. (When $H$ satisfies the strong Lipschitz condition as in [2], this joint calmness can be shown to be equivalent to the one in the theorem.)

\section{Proof of Theorem 2.1.}

Step 1. Reduction to the free endpoint case. We now show how to reduce to the case in which $D=R^{n}$. This hinges upon showing that for some $m, z$ solves the new problem $(\mathrm{Q})$ of minimizing $J(x)+m d_{D}(x(b))$ over the arcs $x$ lying in $\Omega$ and satisfying $x(a) \in C$. If this is false, there is for each positive integer $i$ such an arc $x_{i}$ satisfying

$$
J\left(x_{i}\right)+i d_{D}\left(x_{i}(b)\right)<J(z)=V(0)
$$

Let $d_{i}$ in $D$ satisfy

$$
d_{D}\left(x_{i}(b)\right)=\left|d_{i}-x_{i}(b)\right|,
$$

and set $\alpha_{i}:=x_{i}(b)-d_{i}$. Then $x_{i}$ is feasible for the problem $\left(\mathrm{P}_{\alpha}\right)$ corresponding to $\alpha=\alpha_{i}$, and the above gives

$$
V\left(\alpha_{i}\right) \leq J\left(x_{i}\right)<V(0)-i d_{D}\left(x_{i}(b)\right)=V(0)-i\left|\alpha_{i}\right| .
$$

This implies

$$
\liminf _{\alpha \rightarrow 0} \frac{V(\alpha)-V(0)}{|\alpha|}=-\infty,
$$

contradicting the calmness hypothesis.

Thus $z$ solves the free endpoint problem (Q). However, the assertions of the theorem applied to $(\mathrm{Q})$ (which is automatically calm) imply those for $(\mathrm{P})$. To see this, only the transversality at $b$ needs to be studied, since the other assertions are identical. The transversality for (Q) gives $-p(b) \in \partial\left\{f+m d_{D}\right\}(z(b))$. The right side is contained in $\partial f(z(b))+m \partial d_{D}(z(b))$ by $[3,2.3 .3]$, which is itself contained in $\partial f(z(b))+N_{D}(z(b))$ by $[3,2.4 .2]$. The transversality condition for (P) ensues.

We now continue the proof of the theorem supposing that $D$ is $R^{n}$.

Step 2. A family of auxiliary Lagrangians and Hamiltonians. We shall construct auxiliary Lagrangians $L_{M}$ with special properties, and later certain problems in terms of $L_{M}$, whose solutions will converge to $z$ as $M \rightarrow \infty$ (along a sequence). Let us define, for any $M \geq 1$,

$$
k_{M}(t):=\left|z^{\prime}(t)\right|+M+h_{p}(t, M)
$$


where $h_{p}$ has been previously defined. Note that $k_{M}(\cdot)$ belongs to $L^{1}$. We set

$$
H_{M}(t, x, p):=\min _{|q| \leq M}\left\{H(t, x, q)+k_{M}(t)|p-q|\right\} .
$$

It follows readily that $H_{M}(t, x, \cdot)$ is convex [3, lemma, p. 237]. We let $L_{M}$ be the associated Lagrangian:

$$
L_{M}(t, x, v):=\sup _{p}\left\{p \cdot v-H_{M}(t, x, p)\right\}
$$

( $L_{M}$ may be thought of as $+\infty$ for $(t, x)$ lying outside $\Omega$ ). Our hypotheses imply readily (see [6]) that $L_{M}$ is $L \times B$-measurable, and lower semicontinuous in $(x, v)$. Further properties are given by the following:

PROPOSITION 3.1. (i) $H_{M}$ satisfies the strong Lipschitz condition, and coincides with $H$ for $|p| \leq M$.

(ii) $L_{M}(t, x, v)$ is given by

$$
\sup _{p} \sup _{|q| \leq M}\left\{p \cdot v-H(t, x, q)-k_{M}(t)|q-p|\right\}
$$

and is finite iff $|v| \leq k_{M}(t)$.

(iii) There is an integrable function $\varphi_{1}(\cdot)$ such that for all $M \geq 1$,

$$
L_{M}(t, x, v) \geq \varphi_{1}(t) \quad \text { for }(t, x) \in \Omega, v \in R^{n} .
$$

(iv) $L_{M}\left(t, z(t), z^{\prime}(t)\right) \leq L\left(t, z(t), z^{\prime}(t)\right)$ a.e.

PROOF. The strong Lipschitz condition for $H_{M}$ is easily proven directly from the fact that $H(t, \cdot, \cdot)$ is locally Lipschitz, together with (H2). If $|p|,|q| \leq M$, we have

$$
H(t, x, q)+k_{M}(t)|p-q| \geq H(t, x, p)
$$

by definition of $k_{M}(t)$, which implies the remaining assertion in (i).

The expression for $L_{M}$ in (ii) follows by substituting the defining expression for $H_{M}$ into that of $L_{M}$. If $|v| \leq k_{M}(t)$, the quantity in braces is easily seen to be majorized by $k_{M}(t)|q|-H(t, x, q)$, whence $L_{M}(t, x, v)$ is finite. When $|v|$ exceeds $k_{M}(t)$, it is easy to see that (for any $q$ ) the supremum in $p$ is $+\infty$, whence $L_{M}(t, x, v)=+\infty$.

If we set $p=q=0$ in (ii) we derive

$$
L_{M}(t, x, v) \geq-H(t, x, 0),
$$

which implies (iii). We turn finally to (iv). Let $p$ and $q$ be fixed. We calculate

$$
p \cdot z^{\prime}(t)-q \cdot z^{\prime}(t) \leq\left|z^{\prime}(t)\right||p-q| \leq k_{M}(t)|p-q| .
$$

This implies that the supremum in (ii), for $v=z^{\prime}(t)$ and for $q$ given, is attained for $p=q$. We conclude

$$
\begin{aligned}
L_{M}\left(t, z, z^{\prime}\right) & =\sup _{|q| \leq M}\left\{q \cdot z^{\prime}-H(t, z, q)\right\} \\
& \leq \sup _{q}\left\{q \cdot z^{\prime}-H(t, z, q)\right\}=L\left(t, z, z^{\prime}\right),
\end{aligned}
$$

which is (iv). Q.E.D. 
Step 3. A family of optimization problems. For each $M \geq 1$, we define a problem of Bolza $\left(P_{M}\right)$ as follows: to minimize

$$
J_{M}(x):=f(x(b))+\int_{a}^{b} L_{M}\left(t, x(t), x^{\prime}(t)\right) d t+\frac{1}{2} \int_{a}^{b}|x(t)-z(t)|^{2} d t
$$

over all $\operatorname{arcs} x$ in $A$ satisfying $x(a) \in C$. Recall that $L_{M}$ is $+\infty$ when $(t, x)$ is outside $\Omega$, so that the constraint $(t, x(t)) \in \Omega$ is implicit. With this observation, it is easy to see that the compactness criterion of Rockafellar applies to give the existence of a solution $x_{M}$ to $\left(P_{M}\right)$. Since $z$ is itself feasible for $\left(P_{M}\right)$, we have (in light of Proposition 3.1(iv))

$$
J_{M}\left(x_{M}\right) \leq J_{M}(z) \leq J(z) .
$$

Proposition 3.2. The set $\left\{x_{M}: M \geq 1\right\}$ is relatively weakly compact in $A$.

Proof. We shall define a Lagrangian $L_{0}$ and associated Hamiltonian $H_{0}$ satisfying the basic hypotheses and basic growth condition, and such that $L_{0} \leq L_{M}$ for every $M \geq 1$. Then, for each $M$,

$$
J_{0}\left(x_{M}\right) \leq J_{M}\left(x_{M}\right) \leq J(z)
$$

where $J_{0}(x)$ is given by

$$
f(x(b))+\int_{a}^{b} L_{0}\left(t, x, x^{\prime}\right) d t
$$

Thus the set in the statement of the proposition will be a subset of the set $\{x \in$ $\left.A: J_{0}(x) \leq J(z)\right\}$, which is weakly compact by Rockafellar's semicontinuity theorem $(\S 2)$, and the result will follow.

We define $H_{0}$ first, via

$$
H_{0}(t, x, p):=\sup _{M} H_{M}(t, x, p)=\sup _{M} \inf _{|q| \leq M}\left\{H(t, x, q)+k_{M}(t)|p-q|\right\}
$$

for $(t, x) \in \Omega$, and we set $H_{0}=-\infty$ outside $\Omega$. Note that by Proposition 1 , $H_{M}(t, x, p)=H(t, x, p)$ for $M \geq|p|$, so that $H_{0}(t, x, p)$ is also expressible as $\sup _{M \leq|p|} H_{M}(t, x, p)$. For each $M$ with $M \leq|p|$ we have (by taking $q=0$ in the infimum defining $H_{M}$ )

$$
\begin{aligned}
H_{M}(t, x, p) & \leq H(t, x, 0)+k_{M}(t)|p| \\
& \leq H(t, x, 0)+k_{|p|}(t)|p| \leq h(t, 0)+k_{|p|}(t)|p| .
\end{aligned}
$$

It follows that the last term, which defines an integrable function on $[a, b]$, also majorizes $H_{0}(t, x, p)$, which therefore satisfies the basic growth condition. It is routine to verify that $H_{0}$ is $L \times B$ measurable.

We define $L_{0}$ as the Lagrangian corresponding to $H_{0}$ :

$$
L_{0}(t, x, v)=\sup _{p}\left\{\langle p, v\rangle-H_{0}(t, x, p)\right\} .
$$

The condition $H_{0} \geq H_{M}$ translates as $L_{0} \leq L_{M}$, and so the proof of Proposition 3.2 is complete. Q.E.D.

In view of Proposition 3.2 we may pick a sequence $M_{i}$ tending to $\infty$ with the property that for some arc $y$ we have

$$
\begin{aligned}
& x_{i}^{\prime} \rightarrow y^{\prime} \quad \text { (weakly in } L^{1} \text { ), } \\
& x_{i} \rightarrow y \quad \text { (uniformly) }
\end{aligned}
$$


where $x_{i}, L_{i}, J_{i}, H_{i}$ will be used to designate $x_{M}, L_{M}, J_{M}, H_{M}$ for $M=M_{i}$. It follows that $y$ itself also lies in $\Omega$ and satisfies $y(a) \in C$. Since $z$ is itself feasible for each $\left(P_{M}\right)$, we have (in light of Proposition 3.1(iv))

$$
J_{i}\left(x_{i}\right) \leq J_{i}(z) \leq J(z)
$$

The terms $J_{i}\left(x_{i}\right)$ are also bounded below (by Proposition 3.1(iii)), so there is no loss of generality in supposing that $\lim _{i \rightarrow \infty} J_{i}\left(x_{i}\right)$ exists (finitely), as well as $\lim _{i \rightarrow \infty} \int_{a}^{b} L_{i}\left(t, x_{i}, x_{i}^{\prime}\right) d t$.

PROPOSITION 3.3.

$$
\int_{a}^{b} L\left(t, y, y^{\prime}\right) d t \leq \lim _{i \rightarrow \infty} \int_{a}^{b} L_{i}\left(t, x_{i}, x_{i}^{\prime}\right) d t
$$

PROOF. The left side is precisely $\int_{a}^{b} \sup _{p}\left\{\left\langle p, y^{\prime}\right\rangle-H(t, y, p)\right\} d t$, which equals (see [6, Proposition 6]) $\sup _{p(\cdot) \in L^{\infty}} \int_{a}^{b}\left\{\left\langle p, y^{\prime}\right\rangle-H(t, y, p)\right\} d t$. For given $p$ in $L^{\infty}$, the integral above is majorized by $\liminf _{i \rightarrow \infty} \int_{a}^{b}\left\{\left\langle p, x_{i}^{\prime}\right\rangle-H_{i}\left(t, x_{i}, p\right)\right\} d t$, by Fatou's lemma, and because $x_{i}^{\prime}$ converges weakly to $y^{\prime}$ in $L^{1}$. We deduce

$$
\begin{aligned}
\int_{a}^{b} L\left(t, y, y^{\prime}\right) d t & \leq \sup _{p \in L^{\infty}} \liminf _{i \rightarrow \infty} \int_{a}^{b}\left\{\left\langle p, x_{i}^{\prime}\right\rangle-H_{i}\left(t, x_{i}, p\right)\right\} d t \\
& \leq \limsup _{i \rightarrow \infty} \sup _{p \in L^{\infty}} \int_{a}^{b}\left\{\left\langle p, x_{i}^{\prime}\right\rangle-H_{i}\left(t, x_{i}, p\right)\right\} d t \\
& =\limsup _{i \rightarrow \infty} \int_{a}^{b} \sup _{p}\left\{\left\langle p, x_{i}^{\prime}\right\rangle-H_{i}\left(t, x_{i}, p\right)\right\} d t \\
& =\lim _{i \rightarrow \infty} \int_{a}^{b} L_{i}\left(t, x_{i}, x_{i}^{\prime}\right) d t
\end{aligned}
$$

as required. Q.E.D.

In view of Proposition 3.3, we have

$$
J(y)+\frac{1}{2} \int_{a}^{b}|y(t)-z(t)|^{2} d t \leq \lim _{i \rightarrow \infty} J_{i}\left(x_{i}\right) \leq J(z) .
$$

It follows that $y=z$, since otherwise $y \in \Omega$ would be strictly better for $P$ than the solution $z$ itself. In consequence, for $i$ sufficiently large, the arc $x_{i}$ lies in the interior of the tube $\Omega$, so that $x_{i}$ is a (strong) local solution to the problem of minimizing $J_{i}(x)$ subject to $x(a) \in C$. Since $H_{i}$ satisfies the strong Lipschitz condition (Proposition 3.1(i)), the necessary conditions of [3, Theorem 4.2.2] are available. These affirm the existence of an arc $p_{i}$ such that

$$
\begin{aligned}
& \left(-p_{i}^{\prime}, x_{i}^{\prime}\right) \in \partial H_{i}\left(t, x_{i}, p_{i}\right)-\left(x_{i}-z, 0\right) \quad \text { a.e., } \\
& H_{i}\left(t, x_{i}, p_{i}\right)=p_{i} \cdot x_{i}^{\prime}-L_{i}\left(t, x_{i}, x_{i}^{\prime}\right) \quad \text { a.e., } \\
& p_{i}(a) \in N_{C}\left(x_{i}(a)\right), \quad-p_{i}(b) \in \partial f\left(x_{i}(b)\right) .
\end{aligned}
$$

The last relation gives a bound on $\left|p_{i}(b)\right|$ independent of $i$ (namely, the Lipschitz rank $K_{f}$ of $f$ ). For $i$ sufficiently large, we have $M_{i}>K_{f}$, so that for $t$ near $b$ we have $\left|p_{i}(t)\right|<M_{i}$. Let $S_{i}$ be the largest subinterval $(\tau, b]$ such that this inequality holds on $(\tau, b]$. Then by Proposition 3.1(i) we have

$$
\left(-p_{i}^{\prime}, x_{i}^{\prime}\right) \in \partial H\left(t, x_{i}, p_{i}\right)-\left(x_{i}-z, 0\right) \text { a.e. in } S_{i} .
$$


We now invoke the condition ( $\mathrm{H} 1)$ on $H$ to derive from this

$$
\begin{aligned}
\left|p_{i}^{\prime}\right| & \leq k_{1}(t)\left[1+\left|p_{i}\right|\right]+c_{1}\left[1+\left|p_{i}\right|\right]\left|x_{i}^{\prime}\right|+c_{2}\left|H\left(t, x_{i}, p_{i}\right)\right|+\left|x_{i}-z\right| \\
& \leq\left[1+\left|p_{i}\right|\right]\left[k_{1}(t)+c_{1}\left|x_{i}^{\prime}\right|\right]+c_{2}\left|p_{i}\right|\left|x_{i}^{\prime}\right|+\left|L_{i}\left(t, x_{i}, x_{i}^{\prime}\right)\right|+\left|x_{i}-z\right| .
\end{aligned}
$$

From Proposition 3.2 we may deduce the existence of a constant $c_{3}$ such that for all $i$,

$$
\int_{a}^{b}\left|x_{i}^{\prime}(t)\right| d t \leq c_{3}
$$

It is clear from this that once the lemma below is established, a straightforward application of Gronwall's lemma to the differential inequality above leads to a bound on $p_{i}$ independent of $i$ :

$$
\left|p_{i}(t)\right| \leq c_{4} \quad \text { for } t \text { in } S_{i} .
$$

Then for all $i$ large we have $M_{i}>c_{4}$ and hence $S_{i}=[a, b]$, so that

$$
\left(-p_{i}^{\prime}, x_{i}^{\prime}\right) \in \partial H\left(t, x_{i}, p_{i}\right)-\left(x_{i}-z, 0\right) \quad \text { a.e. on }[a, b] \text {. }
$$

Recall that $x_{i}-z$ converges uniformly to zero. An application of $[3,3.1 .7]$ then yields a subsequence of $p_{i}$ converging to an arc $p$ satisfying all the required conditions. Here is the missing result.

LEMMA. There exists a number $N$ such that

$$
\int_{a}^{b}\left|L_{i}\left(t, x_{i}, x_{i}^{\prime}\right)\right| d t \leq N \quad \text { for all } i .
$$

To see this, let $\varphi_{1}$ be the function put forward in Proposition 3.1, and note

$$
\begin{aligned}
\int_{a}^{b}\left|L_{i}\left(t, x_{i}, x_{i}^{\prime}\right)\right| d t & \leq \int_{a}^{b}\left|L_{i}\left(t, x_{i}, x_{i}^{\prime}\right)-\varphi_{1}\right| d t+\int_{a}^{b}\left|\varphi_{1}(t)\right| d t \\
& =\int_{a}^{b}\left[L_{i}\left(t, x_{i}, x_{i}^{\prime}\right)-\varphi_{1}\right] d t+\left\|\varphi_{1}\right\|_{1} .
\end{aligned}
$$

The right side converges, whence the required conclusion. Q.E.D.

4. Necessary conditions without calmness. In the absence of a constraint qualification such as calmness, it is well known from standard examples in the calculus of variations and optimal control theory that the conclusions of Theorem 2.1 cannot hold, that some possibility of an "abnormal" form of the necessary conditions must be admitted. Roughly speaking, abnormal necessary conditions are obtained when a scalar multiplier associated to the objective functional vanishes; see [3] for appropriate versions of this in mathematical programming and control theory. Based on experience and analogy, we are led to expect that in abnormal necessary conditions for the Bolza problem $(\mathrm{P})$, there would enter a term related to the objective integrand $\lambda L$, for some nonnegative $\lambda$. The associated Hamiltonian $H_{\lambda}$ would then be given by

$$
H_{\lambda}(t, x, p):=\sup _{v}\{\langle p, v\rangle-\lambda L(t, x, v)\} .
$$

The usual Hamiltonian $H$ would correspond to $H_{1}$. The difficulty is that the abnormal case $\lambda=0$ gives rise to a Hamiltonian $H_{0}$ rather devoid of useful content (i.e., the indicator of the set $\{0\}$ ). In this section we will deal with this difficulty by 
defining suitably $\partial H_{0}$, rather than $H_{0}$ itself. The approach is based on the identity $H_{\lambda}(t, x, p)=\lambda H(t, x, p / \lambda)$ for $\lambda>0$. By taking derivatives in the right-hand expression before carefully letting $\lambda$ decrease to 0 , we can obtain a closed convex set leading to meaningful necessary conditions, the first such for the general problem of Bolza in the absence of a constraint qualification (calmness). We denote the set so obtained by $\partial H_{0}(t, x, p)$, but emphasize that $\partial H_{0}$ is now to be understood as a single symbol which describes a special process of obtaining derivative information from $H_{\lambda}$ as $\lambda$ goes to zero. Of course, this symbol also unifies the notation in the theorem to follow. We continue to posit the basic hypotheses, the basic growth condition, and (H1) (H2). Let $(t, x)$ by any point in Int $\Omega$.

DEFINITION. Let $k: R^{n} \times R^{n} \rightarrow R \cup\{ \pm \infty\}$ be given by

$$
k(\alpha, \beta):=\lim _{\lambda \downarrow 0} \limsup _{\substack{(\tilde{x}, \tilde{p}) \rightarrow(x, p) \\ \varepsilon \downarrow 0}} \inf _{\left|\left(\alpha^{\prime}, \beta^{\prime}\right)-(\alpha, \beta)\right| \leq \lambda} H^{\circ}\left(t, \tilde{x}, \tilde{p} / \varepsilon ; \varepsilon \alpha^{\prime}, \beta^{\prime}\right)
$$

where $H^{\circ}$ designates the generalized directional derivative in $(x, p)$ [3, p. 25]. As a function of $(\alpha, \beta), H^{\circ}(t, x, p ; \alpha, \beta)$ is positively homogeneous, subadditive, and lower semicontinuous, and it can be shown that $k$ inherits these properties. We define

$$
\partial H_{0}(t, x, p):=\{(q, v):\langle(q, v),(\alpha, \beta)\rangle \leq k(\alpha, \beta) \text { for all }(\alpha, \beta)\} .
$$

If $k(\alpha, \beta)$ is $-\infty$ for some value of $(\alpha, \beta)$, then clearly $\partial H_{0}(t, x, p)$ is empty; otherwise it is a nonempty closed convex set whose support function is $k[3,2.1 .4]$.

As we now see, $\partial H_{0}(t, x, p)$ is closely related to the set $\Gamma(t, x, p)$ defined by

$$
\operatorname{cl} \operatorname{co}\left\{\lim \left[\varepsilon_{i} \phi_{i}, \psi_{i}\right]:\left(\varphi_{i}, \psi_{i}\right) \in \partial H\left(t, x_{i}, p_{i} / \varepsilon_{i}\right),\left(x_{i}, p_{i}\right) \rightarrow(x, p), \varepsilon_{i} \downarrow 0\right\} .
$$

Proposition 4.1. We always have $\partial H_{0}(t, x, p) \supset \Gamma(t, x, p)$, and if every sequence $\left[\varepsilon_{i} \varphi_{i}, \psi_{i}\right]$ as in the definition of $\Gamma$ is bounded, we have equality.

PROOF. We have observed that $k$ is the support function of $\partial H_{0}$ when the latter is nonempty; thus $(q, v)$ lies in $\partial H_{0}$ iff $k(\alpha, \beta)$ majorizes $(\alpha, \beta) \cdot(q, v)$ for all $(\alpha, \beta)$. Let $\gamma=\lim \left[\varepsilon_{i} \varphi_{i}, \psi_{i}\right]$ be a point of the type described in defining $\Gamma$. To prove the required inclusion, it suffices to show that each such $\gamma$ belongs to $\partial H_{0}$, which is equivalent to verifying that $k(\alpha, \beta)$ majorizes $\gamma \cdot(\alpha, \beta)$ for each $(\alpha, \beta)$. To see this, let $\lambda$ be a positive number and let $\left(\alpha^{\prime}, \beta^{\prime}\right)$ be a point within $\lambda$ of $(\alpha, \beta)$. Then

$$
\begin{aligned}
H^{\circ}\left(t, x_{i}, p_{i} / \varepsilon_{i} ; \varepsilon_{i} \alpha^{\prime}, \beta^{\prime}\right) & \geq\left[\varphi_{i}, \psi_{i}\right] \cdot\left[\varepsilon_{i} \alpha^{\prime}, \beta^{\prime}\right] \\
& \geq\left[\varepsilon_{i} \varphi_{i}, \psi_{i}\right] \cdot[\alpha, \beta]-\lambda\left|\left(\varepsilon_{i} \varphi_{i}, \psi_{i}\right)\right| .
\end{aligned}
$$

This leads easily to the requird inequality. Now let the extra hypothesis hcld, and let any $(\alpha, \beta)$ be given. For certain sequences $x_{i}, p_{i}, \varepsilon_{i}, \alpha_{i}$ and $\beta_{i}$ converging to $x, p, 0, \alpha$, and $\beta$ respectively, we have

$$
k(\alpha, \beta)=\lim H^{\circ}\left(t, x_{i}, p_{i} / \varepsilon_{i} ; \varepsilon \alpha_{i}, \beta_{i}\right)=\lim \left[\varepsilon_{i} \alpha_{i}, \beta_{i}\right] \cdot\left[\varphi_{i}, \psi_{i}\right]
$$

for certain points $\left(\varphi_{i}, \psi_{i}\right)$ belonging to $\partial H\left(t, x_{i}, p_{i} / \varepsilon_{i}\right)$ (by $\left.[\mathbf{3}, 2.1 .5]\right)$. For a suitable subsequence $\left(\varepsilon_{i} \varphi_{i}, \psi_{i}\right)$ converges to an element $\gamma$ of $\Gamma(t, x, p)$, whence $k(\alpha, \beta)$ equals $\gamma \cdot(\alpha, \beta)$, proving that $\partial H_{0}(t, x, p)$ is contained in $\Gamma(t, x, p)$. Q.E.D.

The following gives a simple criterion assuring that $\partial H_{0}$ is empty, a superlinear growth condition on $H$ in the $p$ variable. 
Proposition 4.2. Let $H(t, x, p)$ be locally Lipschitz in $(x, p)$ and satisfy the following condition for some $p \neq 0$ :

$$
\lim _{\substack{x \rightarrow x \\ \tilde{p} \rightarrow p \\ \lambda \rightarrow \infty}} \frac{H(t, \tilde{x}, \lambda \tilde{p})}{\lambda}=\infty .
$$

Then $\partial H_{0}(t, x, p)=\varnothing$.

ProOF. We shall prove this by showing that $k(0,-p)$ is $-\infty$. Let $\lambda_{i}$ be a sequence decreasing to 0 , and choose $\left(x_{i}, p_{i}\right)$ in $(x, p)+\lambda_{i} B$ and $\varepsilon_{i}$ in the interval $\left(0, \lambda_{i}\right)$ such that

$$
\begin{aligned}
\inf _{(\alpha, \beta) \in(0,-p)+\lambda_{i} B} & H^{\circ}\left(t, x_{i}, p_{i} / \varepsilon_{i} ; \varepsilon_{i} \alpha, \beta\right)+\lambda_{i} \\
& \geq \lim _{\substack{(\tilde{x}, \tilde{p}) \rightarrow(x, p) \\
\varepsilon \downarrow 0}} \inf _{\substack{(\alpha, \beta) \in(0,-p)+\lambda_{i} B\\
}} H^{\circ}(t, \tilde{x}, \tilde{p} / \varepsilon ; \varepsilon \alpha, \beta) .
\end{aligned}
$$

It follows then that we have

$$
\liminf _{i \rightarrow \infty} H^{\circ}\left(t, x_{i}, p_{i} / \varepsilon_{i} ; 0,-p_{i}\right) \geq k(0,-p) .
$$

For some element $\left(\varphi_{i}, \psi_{i}\right)$ of $\partial H\left(t, x_{i}, p_{i} / \varepsilon_{i}\right)$ we have

$$
H^{\circ}\left(t, x_{i}, p_{i} / \varepsilon_{i} ; 0,-p_{i}\right)=\left\langle\left(0,-p_{i}\right),\left(\varphi_{i}, \psi_{i}\right)\right\rangle=-\left\langle p_{i}, \psi_{i}\right\rangle,
$$

and the convexity of $H\left(t, x_{i}, \cdot\right)$ gives

$$
H\left(t, x_{i}, 0\right)-H\left(t, x_{i}, p_{i} / \varepsilon_{i}\right) \geq-\left\langle p_{i}, \psi_{i}\right\rangle / \varepsilon_{i} ;
$$

whence

$$
-\left\langle p_{i}, \psi_{i}\right\rangle \leq \varepsilon_{i} h(t, 0)-\varepsilon_{i} H\left(t, x_{i}, p_{i} / \varepsilon_{i}\right) .
$$

The last term goes to $-\infty$ as $i \rightarrow \infty$, by hypothesis, whence $k(0,-p)=-\infty$. Q.E.D.

THEOREM 4.1. If $z$ solves $(\mathrm{P})$, where all the hypotheses of Theorem 2.1 hold except calmness, then there exists an arc $p$ and a scalar $\lambda$ equal to 0 or 1 such that $\lambda+\|p\|_{\infty}>0$ and

$$
\begin{gathered}
\left(-p^{\prime}(t), z^{\prime}(t)\right) \in \partial H_{\lambda}(t, z(t), p(t)) \quad \text { a.e. } \\
p(a) \in \operatorname{cl} N_{C}(z(a)), \quad-p(b) \in \lambda \partial f(z(b))+\operatorname{cl} N_{D}(z(b)) .
\end{gathered}
$$

PROOF OF THE THEOREM. We define a family of parametrized problems $\left(\mathrm{Q}_{\alpha}\right)$ :

$$
\text { minimize } J(x)+\int_{a}^{b}|x-z|^{2} d t: x \in \mathcal{A}, x(a) \in C, x(b) \in D+\alpha,
$$

and we let $W(\alpha)$ be the associated value function. Note that $z$ is the unique solution to $\left(\mathrm{Q}_{0}\right)$. It follows easily from Rockafellar's semicontinuity theorem (§2) that $W$ is lower semicontinuous, and that $\left(\mathrm{Q}_{\alpha}\right)$ admits a solution whenever $W(\alpha)$ is finite. It is known (Rockafellar [7]) that $W$ admits proximal subgradients arbitrarily near 0 . More precisely, there exist points $\alpha_{i}$ converging to 0 such that $W\left(\alpha_{i}\right)$ converges to $W(0)$ and such that, for some vector $\zeta_{i}$ and for some positive scalar $\varepsilon_{i}$, one has

$$
W(\alpha)-W\left(\alpha_{i}\right) \geq\left\langle\zeta_{i}, \alpha-\alpha_{i}\right\rangle-\varepsilon_{i}\left|\alpha-\alpha_{i}\right|^{2}
$$


for $\alpha$ near $\alpha_{i}$. Now let $x_{i}$ be a solution to $\left(\mathrm{Q}_{\alpha}\right)$ for $\alpha=\alpha_{i}$. It follows once more from the semicontinuity theorem that $\left\{x_{i}\right\}$ is relatively weakly compact in $A$, so we may select a subsequence (we do not relabel) converging to an element $x_{0}$ of $A$. Clearly, $x_{0}$ is feasible for $\left(Q_{0}\right)$, and semicontinuity yields

$$
J\left(x_{0}\right)+\int_{a}^{b}\left|x_{0}-z\right|^{2} d t \leq \lim W\left(\alpha_{i}\right)=W(0)=J(z),
$$

whence $x_{0}=z$, and $x_{i}$ satisfies $\left\|x_{i}-z\right\|_{\infty}<\varepsilon_{0}$ for $i$ large.

Fix $i$ large. We denote by $d_{i}$ an element of $D$ having the property that $x_{i}(b)=$ $d_{i}+\alpha_{i}$. Now let $x$ be any arc in $\Omega$ satisfying $x(a) \in C$, and let $d$ be any element of $D$. Observe that $x(b)$ lies in $D+\alpha$ where $\alpha:=x(b)-d$. If $x(b)$ is near $x_{i}(b)$ and $d$ is near $d_{i}$, then the point $\alpha$ is near $\alpha_{i}$, so the proximal subgradient inequality may be invoked to obtain

$$
\begin{aligned}
J(x)+\int_{a}^{b}|x-z|^{2} d t \geq & W(\alpha) \geq W\left(\alpha_{i}\right)+\left\langle\zeta_{i}, \alpha-\alpha_{i}\right\rangle-\varepsilon_{i}\left|\alpha-\alpha_{i}\right|^{2} \\
= & J\left(x_{i}\right)+\int_{a}^{b}\left|x_{i}-z\right|^{2} d t+\left\langle\zeta_{i}, x(b)-x_{i}(b)\right\rangle \\
& +\left\langle\zeta_{i}, d_{i}-d\right\rangle-\varepsilon_{i}\left|x(b)-d-x_{i}(b)+d_{i}\right|^{2}
\end{aligned}
$$

Fix $x=x_{i}$ in this relation. We obtain

$$
\left\langle\varsigma_{i}, d_{i}-d\right\rangle-\varepsilon_{i}\left|d_{i}-d\right|^{2} \leq 0 \text { for } d_{i} \text { near } D,
$$

which implies $\zeta_{i} \in-N_{D}\left(d_{i}\right)$. Now fix $d=d_{i}$; then we deduce that $x_{i}$ is a local minimum for the (free endpoint) problem of minimizing

$$
-\left\langle\zeta_{i}, x(b)\right\rangle+\varepsilon_{i}\left|x(b)-x_{i}(b)\right|^{2}+J(x)+\int_{a}^{b}|x-z|^{2} d t
$$

over the $\operatorname{arcs} x$ satisfying $x(a) \in C$. This is a problem to which Theorem 2.1 applies; we deduce the existence of an $\operatorname{arc} p_{i}$ such that

$$
\begin{aligned}
& \left(-p_{i}^{\prime}, x_{i}^{\prime}\right) \in \partial H\left(t, x_{i}, p_{i}\right)-2\left(x_{i}-z, 0\right) \text { a.e., } \\
& -p_{i}(b) \in-\zeta_{i}+\partial f\left(x_{i}(b)\right) \subset N_{D}\left(x_{i}(b)\right)+\partial f\left(x_{i}(b)\right), \\
& p_{i}(a) \in N_{C}\left(x_{i}(a)\right) .
\end{aligned}
$$

The rest of the proof consists of passing to the limit; the case in which $\zeta_{i}$ is bounded will lead to normal conclusions $(\lambda=1)$, otherwise we will get abnormal ones $(\lambda=0)$.

We note that the weak Lipschitz condition (H1) implies the differential inequality

$$
\left|p_{i}^{\prime}\right| \leq\left\{1+\left|p_{i}\right|\right\}\left\{k_{1}(t)+c_{1}\left|x_{i}^{\prime}\right|\right\}+c_{2}\left|H\left(t, x_{i}, p_{i}\right)\right|+2\left|x_{i}-z\right| .
$$

Case 1. $\left\{\varsigma_{i}\right\}$ is bounded.

In this case it follows that $p_{i}(b)$ is bounded. Almost exactly as in the last step of the proof of Theorem 2.1, we combine this fact with the differential inequality above via Gronwall's lemma to obtain a bound on $\|p\|_{\infty}$. Once armed with this, the same sequential compactness of the Hamiltonian inclusion invoked in the proof of Theorem 2.1 leads to the required $\operatorname{arc} p$, with $\lambda=1$.

Case 2. $\left\{\varsigma_{i}\right\}$ unbounded. 
We may suppose $\left|\zeta_{i}\right|>1$ for large $i$. Let us define the arc $q_{i}$ via $q_{i}=p_{i} /\left|\zeta_{i}\right|$. The differential inequality for $p_{i}$ implies

$$
\left|q_{i}^{\prime}\right| \leq\left\{1+\left|q_{i}\right|\right\}\left\{k_{1}(t)+c_{1}\left|x_{i}^{\prime}\right|\right\}+c_{2}\left|H\left(t, x_{i}, p_{i}\right)\right|\left|\zeta_{i}\right|^{-1}+2\left|x_{i}-z\right| .
$$

We have

$$
H\left(t, x_{i}, p_{i}\right)=\left\langle p_{i}, x_{i}^{\prime}\right\rangle-L\left(t, x_{i}, x_{i}^{\prime}\right)
$$

so

$$
\left|H\left(t, x_{i}, p_{i}\right)\right|\left|s_{i}\right|^{-1} \leq\left|q_{i}\right|\left|x_{i}^{\prime}\right|+\left|L\left(t, x_{i}, x_{i}^{\prime}\right)\right| q_{i} .
$$

With this observation, we obtain much as before a uniform bound on $\left\|q_{i}\right\|_{\infty}$. (note that $q_{i}(b)$ is bounded by construction; in fact, $\left|q_{i}(b)\right|$ converges to 1$)$. For a suitable subsequence therefore (we do not relabel), we may suppose that $q_{i}$ converges uniformly to an arc $p$, and $q_{i}^{\prime}$ weakly to $p^{\prime}$. Since $|p(b)|=1$, necessarily, we have $\|p\|_{\infty}>0$. We already know that $x_{i}$ converges to $z$, so that we have $p(a) \in \operatorname{cl} N_{C}(z(a)), \quad-p(b) \in \operatorname{cl} N_{D}(z(b))$. We need only verify that a.e., $\left(-p^{\prime}, z^{\prime}\right)$ belongs to $\partial H_{0}(t, z, p)$, which is equivalent to verifying that a.e., $k(\alpha, \beta)$ majorizes $\left[-p^{\prime}, z^{\prime}\right] \cdot[\alpha, \beta]$.

Let $t$ lie in the set of full measure in which $x_{i}^{\prime}(t), p_{i}^{\prime}(t)$ exist and the Hamiltonian inclusion for $\left(x_{i}, p_{i}\right)$ holds for each $i$. Set $\varepsilon_{i}=\left|\zeta_{i}\right|^{-1}$, let $\lambda$ be any positive number, and let $\left(\alpha^{\prime}, \beta^{\prime}\right)$ lie within $\lambda$ of $(\alpha, \beta)$. Then

$$
\begin{aligned}
H^{\circ}\left(t, x_{i}, \frac{q_{i}}{\varepsilon_{i}} ; \varepsilon_{i} \alpha^{\prime}, \beta^{\prime}\right) & \geq\left\langle\left(\varepsilon_{i} \alpha^{\prime}, \beta^{\prime}\right),\left(-p_{i}^{\prime}, x_{i}^{\prime}\right)\right\rangle=\left\langle\left(\alpha^{\prime}, \beta^{\prime}\right),\left(q_{i}^{\prime}, x_{i}^{\prime}\right)\right\rangle \\
& \geq\left\langle(\alpha, \beta),\left(q_{i}^{\prime}, x_{i}^{\prime}\right)\right\rangle-\lambda\left|\left(q_{i}^{\prime}, x_{i}^{\prime}\right)\right|
\end{aligned}
$$

since $\left(-p_{i}^{\prime}, x_{i}^{\prime}\right)$ belongs to $\partial H\left(t, x_{i}, q_{i} / \varepsilon_{i}\right)$ (note that $\left.q_{i} / \varepsilon_{i}=p_{i}\right)$. It follows from the Hamiltonian inclusion and (H2) that a.e. for fixed $t,\left|\left(-q_{i}^{\prime}, x_{i}^{\prime}\right)\right|$ is bounded by a quantity $\theta(t)$. An application of the lemma below shows that provided $t$ belongs to a further set of full measure, we have

$$
\limsup _{i \rightarrow \infty} \inf H^{\circ}\left(t, x_{i}, \frac{q_{i}}{\varepsilon_{i}} ; \varepsilon_{i} \alpha^{\prime}, \beta^{\prime}\right) \geq\left\langle(\alpha, \beta),\left(-p^{\prime}, z^{\prime}\right)\right\rangle-\lambda \theta(t)
$$

where the infimum is over $\left(\alpha^{\prime}, \beta^{\prime}\right)$ within $\lambda$ of $(\alpha, \beta)$. Since $x_{i}(t), q_{i}(t), \varepsilon_{i}$ converge to $z(t), p(t), 0$ respectively, we obtain $k(\alpha, \beta) \geq\left[-p^{\prime}, z^{\prime}\right] \cdot[\alpha, \beta]$ as required.

LEMMA. Let $f_{i}$ be a sequence of functions from $[a, b]$ to $R^{n}$ converging weakly in $L^{1}$ to $f$. Then for almost every $t$ one has

$$
\limsup _{i \rightarrow \infty}\left\langle v, f_{i}(t)\right\rangle \geq\langle v, f(t)\rangle \quad \text { for all } v \text { in } R^{n} .
$$

To see this, suppose the conclusion is false on a set $A$ of positive measure. Then we can find a measurable function $v(t)$ defined on $A$ such that

$$
\limsup _{i \rightarrow \infty}\left\langle v(t), f_{i}(t)\right\rangle<\langle v(t), f(t)\rangle, \quad t \in A,
$$


and by homogeneity we can also arrange for $v$ to lie in $L^{\infty}$ and satisfy $\left|\left\langle v(t), f_{i}(t)\right\rangle\right| \leq$ 1. Then

$$
\begin{aligned}
0 & =\limsup _{i \rightarrow \infty} \int_{A}\left\langle v(t), f_{i}(t)-f(t)\right\rangle d t \\
& \left(\text { since } f_{i} \text { converges weakly to } f\right) \\
\leq & \int_{A} \limsup _{i \rightarrow \infty}\left\langle v(t), f_{i}(t)-f(t)\right\rangle d t<0
\end{aligned}
$$

(by Fatou's lemma).

This contradiction proves the lemma and completes the proof of the theorem. Q.E.D.

REMARK. Let $V$ be the value function of $\S 2$, and suppose that $z$ is a (locally) unique solution to the Bolza problem. Then any bounded sequence of proximal subgradients of $V$ as above can be used as in the proof of the theorem to produce necessary conditions in normal form. A necessary and sufficient condition for the existence of such a sequence is $\partial V(0) \neq \varnothing$, so that this condition is now seen to be a constraint qualification assuring that normal necessary conditions hold. Calmness is a stronger condition which implies the nonemptiness of $\partial V(0)$.

We now proceed to illustrate the use of the theorem in two well-known contexts. The first example will confirm that we recover the appropriate necessary conditions in the case of optimal control problems, and the second example will add to the regularity results of [5] by providing an additional set of hypotheses under which the basic problem in the calculus of variations has a solution with bounded derivative.

Let $U$ be a compact subset of $R^{m}$, and let $\varphi$ and $g$ by $C^{1}$ mappings from $R^{n} \times U$ to $R^{n}$ and $R$ respectively. Given two closed subsets $C$ and $D$ of $R^{n}$, the standard optimal control problem we consider is that of minimizing $\int_{a}^{b} g(x(t), u(t)) d t$ over the $\operatorname{arcs} x$ (states) and measurable function $u$ (controls) satisfying

$$
x^{\prime}(t)=\varphi(x(t), u(t)) \text { a.e., } \quad u(t) \in U \text { a.e., } \quad x(a) \in C, \quad x(b) \in D .
$$

We assume that the set $\{[\varphi(x, u), g(x, u)+\delta]: u \in U, \delta \geq 0\}$ is convex for each $x$, a standard hypothesis in existence theory [3, Theorem 5.4.4].

The problem above is equivalent to [3, Theorem 5.4.1] the generalized problem of Bolza in which $L$ and $H$ are given by

$$
\begin{aligned}
L(x, v) & :=\inf \{g(x, u): u \in U, \varphi(x, u)=v\}, \\
H(x, p) & :=\max \{\langle p, \varphi(x, u)\rangle-g(x, u): u \in U\} .
\end{aligned}
$$

It follows easily that these data satisfy the hypotheses of Theorem 4.1. Suppose therefore that $x$ is a solution to the problem (i.e., that for some corresponding control $u,(x, u)$ solves the optimal control problem). According to the theorem, there is an arc $p$ satisfying the transversality conditions $p(a) \in \operatorname{cl} N_{C}(x(a)),-p(a) \in$ $\operatorname{cl} N_{D}(x(b))$ and either $\left(-p^{\prime}, x^{\prime}\right) \in \partial H(x, p)$ a.e. or else $\left(-p^{\prime}, x^{\prime}\right) \in \partial H_{0}(x, p)$ a.e., and in the latter case $p$ is nonzero. The first situation corresponds to standard necessary conditions in normal form as would necessarily be the case in the presence of calmness [3], so let us examine the second case by calculating $\partial H_{0}(x, p)$.

The set $\Gamma$ of Proposition 4.1 is generated by limits of points $[\varepsilon \zeta, \psi]$ where $(\zeta, \psi)$ belongs to $\partial H\left(x^{\prime}, p^{\prime} / \varepsilon\right)$. The set of such $(\zeta, \psi)$ is given by [3, Theorem 2.8.2, 
Corollary 2]

$$
\operatorname{co}\left\{\left[\varphi_{x}\left(x^{\prime}, u^{\prime}\right)^{*} p^{\prime} / \varepsilon-g_{x}\left(x^{\prime}, u^{\prime}\right), \varphi\left(x^{\prime}, u^{\prime}\right)\right]: u^{\prime} \in M\left(x^{\prime}, p^{\prime} / \varepsilon\right)\right\}
$$

where $M(x, p)$ denotes the points $u^{\prime}$ in $U$ at which the maximum defining $H(x, p)$ is attained. It follows that $(\varepsilon \zeta, \psi)$ admits an a priori bound, and that any limit of a series of such points as $x^{\prime} \rightarrow x, p^{\prime} \rightarrow p$ and $\varepsilon \downarrow 0$ lies in the set

$$
\operatorname{co}\left\{\left[\varphi_{x}\left(x, u^{\prime}\right)^{*} p, \varphi\left(x, u^{\prime}\right)\right]: u^{\prime} \in N(x, p)\right\},
$$

where $N(x, p)$ denotes the set of points $u^{\prime}$ in $U$ at which the maximum of $\langle p, \varphi(x, v)\rangle$ over $v$ in $U$ is attained.

It follows from Proposition 4.1 that the last set above is precisely $\partial H_{0}(x, p)$. To see why this leads to the expected sort of condition, define

$$
h_{\lambda}(x, p):=\max \{\langle p, \varphi(x, u)\rangle-\lambda g(x, u): u \in U\},
$$

a function which makes sense for any $\lambda$, including 0 . Then Theorem 4.2 amounts to saying that $p$ satisfies $\left(-p^{\prime}, x^{\prime}\right) \in \partial h_{\lambda}(x, p)$ a.e. for $\lambda$ either 1 or 0 , and that $p$ is nontrivial in the latter case. This is precisely what one would hope to get.

The second special context is that of the basic problem in the calculus of variations, where $C$ and $D$ are singletons and $L$ is a Lagrangian of classical type. To simplify, we again posit smoothness. To be precise, we suppose that $L(t, x, v)$ is measurable in $t$, continuously differentiable in $(x, v)$, and strictly convex in $v$. We further suppose that $L$ satisfies the following growth conditions:

$$
\begin{gathered}
\left|L_{x}(t, x, v)\right| \leq\left[1+\left|L_{v}(t, x, v)\right|\right]\left[m(t)+d_{1}|v|\right]+d_{2}|L(t, x, v)|, \\
e_{1}|v|^{1+r}+e_{2} \leq L(t, x, v) \leq e_{3}|v|^{1+s}+e_{4}
\end{gathered}
$$

where $m(\cdot)$ is summable and all constants are positive.

Tonelli's existence theorem asserts that a solution $x$ to the problem exists. We assert that the solution $x(\cdot)$ is Lipschitz on $[a, b]$, which is a new result (cf. [5]). The proof will follow from analyzing the necessary conditions provided by Theorem 4.1. The example in [4] shows that the conclusion is false in the absence of (4.1). We proceed first to verify the hypotheses of the theorem.

The measurability and lower semicontinuity requirements on $L$ are clearly subsumed by the present hypotheses. By conjugacy and (4.2), $H$ satisfies a global condition

$$
f_{1}|p|^{1+\sigma}+f_{2} \leq H(t, x, p) \leq f_{3}|p|^{1+\tau}+f_{4}
$$

for certain positive constants, which implies the basic growth condition. Let $v^{*}(t, x, p)$ denote the unique $v$ at which the maximum defining $H(t, x, p)$ is achieved:

$$
\max \left\{\langle p, v\rangle-L(t, x, v): v \in R^{n}\right\}=\left\langle p, v^{*}\right\rangle-L\left(t, x, v^{*}\right) .
$$

Then $H(t, x, \cdot)$ is differentiable and $H_{p}(t, x, p)=v^{*}(t, x, p)$.

LEMMA. $v^{*}$ is bounded on bounded subsets of $[a, b] \times R^{n} \times R^{n}$.

To see this, we observe

$$
\sup \left\{\langle p, w\rangle-e_{3}|w|^{1+s}-e_{4}\right\} \leq \sup \{\langle p, w\rangle-L(t, x, w)\}=\left\langle p, v^{*}\right\rangle-L\left(t, x, v^{*}\right) .
$$

If $x, p$ lie in a bounded set this provides

$$
L\left(t, x, v^{*}\right)-\left\langle p, v^{*}\right\rangle \leq M
$$


for some constant $M$, which yields

$$
e_{1}\left|v^{*}\right|^{1+r}+e_{2}-|p|\left|v^{*}\right| \leq M,
$$

proving the lemma.

Let us now verify (H1). It follows from [3, Theorem 2.8.2] that $H(t, \cdot, \cdot)$ is continuously differentiable, with derivative $(\varphi, \psi)$ at $(x, p)$ given by $\varphi=-L_{x}\left(t, x, v^{*}\right), \psi$ $=v^{*}$. Note also that $p$ equals $L_{v}\left(t, x, v^{*}\right)$. In consequence, (H1) amounts to requiring

$$
\left|L_{x}\left(t, x, v^{*}\right)\right| \leq\left\{1+\left|L_{v}\left(t, x, v^{*}\right)\right|\right\}\left\{k_{1}(t)+c_{1}\left|v^{*}\right|\right\}+c_{2}\left|\left\langle p, v^{*}\right\rangle-L\left(t, x, v^{*}\right)\right| .
$$

But the growth hypothesis (4.1) on $L$ gives

$$
\begin{aligned}
\left|L_{x}\left(t, x, v^{*}\right)\right| \leq\{1 & \left.+\left|L_{v}\left(t, x, v^{*}\right)\right|\right]\left[m(t)+d_{1}\left|v^{*}\right|\right] \\
& \left.+d_{2}\left|\left\langle p, v^{*}\right\rangle-L\left(t, x, v^{*}\right)\right|+d_{2}\left|\left\langle p, v^{*}\right\rangle\right|\right\},
\end{aligned}
$$

which implies the required type of condition upon replacing $p$ once again by $L_{v}\left(t, x, v^{*}\right)$. We turn now to $(\mathrm{H} 2)$.

Since $H_{p}(t, x, p)=v^{*}(t, x, p)$ the lemma implies that $h_{p}(\cdot, M)$ is bounded. As for $h_{x}(t, M)$, we observe from (H1) that for each $p$ with $|p| \leq M, H_{x}(t, x, p)$ is bounded above by

$$
\begin{aligned}
\{1+ & M\}\left\{k_{1}(t)+c_{1}\left|v^{*}(t, x, p)\right|\right\}+c_{2}|H(t, x, p)| \\
& \leq\{1+M\}\left\{k_{1}(t)+c_{1}\left|v^{*}(t, x, p)\right|\right\}+c_{2}\left\{f_{3}|p|^{1+\tau}+f_{4}\right\} .
\end{aligned}
$$

Since $v^{*}$ is bounded by the lemma, it follows that $h_{x}(\cdot, M)$ is integrable.

The above confirms the applicability of the theorem. We now wish to show, without an a priori constraint qualification, that the abnormal case $\lambda=0$ can be excluded, which is obviously the case if $\partial H_{0}(t, x, p)=\varnothing$ for $p \neq 0$. But this follows immediately from Proposition 4.2, in view of the growth condition satisfied by $H$. Thus the conclusions of the theorem must hold for $\lambda=1$.

The Hamiltonian inclusion for $\lambda=1$ implies that a.e.

$$
x^{\prime}(t)=H_{p}(t, x(t), p(t))=v^{*}(t, x(t), p(t)),
$$

and so by the lemma $x^{\prime}(\cdot)$ is essentially bounded, which is the desired conclusion.

\section{REFERENCES}

1. F. H. Clarke, The generalized problem of Bolza, SIAM J. Control Optim. 14 (1976), 682-699.

2. __ Eatremal arcs and extended Hamiltonian systems, Trans. Amer. Math. Soc. 231 (1977), 349-367.

3. ___ Optimization and nonsmooth analysis, Wiley Interscience, New York, 1983.

4. F. H. Clarke and R. B. Vinter, On the conditions under which the Euler equation or the maximum principle hold, Appl. Math. Optim. 12 (1984), 73-79.

5. _ Regularity properties of solutions to the basic problem in the calculus of variations, Trans. Amer. Math. Soc. 289 (1985), 73-98.

6. R. T. Rockafellar, Existence theorems for general control problems of Bolza and Lagrange, Adv. in Math. 15 (1975), 312-333.

7. __ Extensions of subgradient calculus with applications to optimization, Nonlinear Anal. 9 (1985), 665-698.

8. V. Zeidan, Sufficient conditions for the generalized problem of Bolza, Trans. Amer. Math. Soc. 275 (1983), 561-586.

Centre de Recherches Mathematiques, Universite de Montreal, C.P. 6128, SuCC. "A", Montreal, Quebec, H3C 3J7, CANADA 